CERN-PPE/97-135

9 October 1997

\title{
Direct Determination of Two-Pion Correlations for $\bar{p} p \rightarrow 2 \pi^{+} 2 \pi^{-}$Annihilation at Rest
}

\section{CPLEAR Collaboration}

A. Angelopoulos ${ }^{1}$, A. Apostolakis ${ }^{1}$, E. Aslanides ${ }^{11}$, G. Backenstoss ${ }^{2}$, P. Bargassa ${ }^{13}$, O. Behnke ${ }^{17}$, A. Benelli ${ }^{9}$, V. Bertin ${ }^{11}$, F. Blanc ${ }^{7,13}$, P. Bloch ${ }^{4}$, P. Carlson ${ }^{15}$, M. Carroll ${ }^{9}$, J. Carvalho ${ }^{5}$, E. Cawley ${ }^{9}$, S. Charalambous ${ }^{16}$, G. Chardin ${ }^{14}$, M.B. Chertok ${ }^{3}$, A. Cody ${ }^{9}$, M. Danielsson ${ }^{15}$, M. Dejardin ${ }^{14}$, J. Derre ${ }^{14}$, A. Ealet ${ }^{11}$, C. Eleftheriadis ${ }^{16}$, I. Evangelou ${ }^{8}$, L. Faravel ${ }^{7}$, R. Ferreira-Marques ${ }^{5}$, W. Fetscher ${ }^{17}$, M. Fidecaro ${ }^{4}$, A. Filipčič ${ }^{10}$, D. Francis ${ }^{3}$, J. Fry ${ }^{9}$, E. Gabathuler ${ }^{9}$, R. Gamet ${ }^{9}$, D. Garreta ${ }^{14}$, H.-J. Gerber ${ }^{17}$, A. Go ${ }^{14}$, A. Haselden ${ }^{9}$, P.J. Hayman ${ }^{9}$, F. Henry-Couannier ${ }^{11}$, R.W. Hollander ${ }^{6}$, E. Hubert ${ }^{11}$, K. Jon-And ${ }^{15}$, P.-R. Kettle ${ }^{13}$, P. Kokkas ${ }^{4}$, R. Kreuger ${ }^{6,13}$, R. Le Gac ${ }^{11}$, F. Leimgruber ${ }^{2}$, A. Liolios ${ }^{16}$, E. Machado ${ }^{5}$, I. Mandić ${ }^{10}$, N. Manthos ${ }^{8}$, G. Marel ${ }^{14}$, M. Mikuž ${ }^{10}$, J. Miller $^{3}$, F. Montanet ${ }^{11}$, A. Muller ${ }^{14}$, T. Nakada ${ }^{13}$, B. Pagels ${ }^{17}$, I. Papadopoulos ${ }^{16}$, P. Pavlopoulos ${ }^{2}$, J. Pinto da Cunha ${ }^{5}$, A. Policarpo ${ }^{5}$, G. Polivka ${ }^{2}$, R. Rickenbach ${ }^{2}$, B.L. Roberts ${ }^{3}$, T. Ruf ${ }^{4}$, L. Sakeliou ${ }^{1}$, P. Sanders ${ }^{9}$, M. Schäfer ${ }^{17}$, L.A. Schaller ${ }^{7}$, T. Schietinger ${ }^{2}$, A. Schopper ${ }^{4}$, A. Soares ${ }^{14}$, L. Tauscher ${ }^{2}$, C. Thibault ${ }^{12}$, F. Touchard ${ }^{11}$, C. Touramanis ${ }^{9}$, F. Triantis ${ }^{8}$, E. Van Beveren ${ }^{5}$, C.W.E. Van Eijk ${ }^{6}$, S. Vlachos ${ }^{2}$, P. Weber ${ }^{17}$, O. Wigger ${ }^{13}$, M. Wolter ${ }^{17}$, C. Yeche ${ }^{14}$, D. Zavrtanik ${ }^{10}$, D. Zimmerman ${ }^{3}$ and M.P. Locher ${ }^{18}$, V.E. Markushin ${ }^{18}$.

\begin{abstract}
We study $\pi \pi$ correlations in the exclusive reaction $\overline{\mathrm{p}} \mathrm{p} \rightarrow 2 \pi^{+} 2 \pi^{-}$at rest measured by the CPLEAR experiment. Avoiding the introduction of an arbitrary reference sample, we analyse differential distributions for equal charge pion pairs removing the phase-space factor event by event. A peak at small relative momenta is most pronounced for large total momentum of the pair. The physical implications of bosonic symmetrization for the properties of the pion source, in particular its radius, are briefly discussed. The two extremes considered are the chaotic Hanbury-BrownTwiss mechanism and the coherent Skyrmion model.
\end{abstract}




\section{Introduction}

Any emission amplitude for identical bosons must be symmetrized. In particular, observable effects can be expected for a pair of pions if their momenta become equal, i.e. for vanishing relative momentum. Bose-Einstein (BE) correlations is the name often assigned to a very specific dynamic picture - the Hanbury-Brown-Twiss (HBT) mechanism [1] linking the two-pion correlation function at small relative momentum to the space-time properties of the pion emitting source, in particular its size [2-4].

The goal of this paper is to study the pion correlations in nucleon-antinucleon annihilation at rest where interesting deviations from the stochastic HBT picture of the BE correlations have been observed [5-10]. In the present paper we concentrate on the pion correlations from the exclusive annihilation channel $2 \pi^{+} 2 \pi^{-}$. The main results of this paper are independent of any model assumptions. In particular, we do not rely on the ratio of correlation functions for pion pairs with equal and unequal signs which often have been used in the literature [5,7-9,11,12], nor do we construct any other reference samples which are known to be a source of ambiguities [13-19]. The constraints originating from energy-momentum conservation in nucleon-antinucleon annihilation at rest are very strong and we shall show that they considerably distort the commonly used correlation functions.

Removing the phase-space factor, event by event, we determine directly for the first time the square of the amplitude for pion-pair emission and the corresponding correlation functions. Our results for the differential distributions confirm that equal charge two-pion correlations do indeed peak at small relative momenta.

In Section 2 we describe the analysis of the single-variable correlation function to make a connection with previous analyses. In Section 3 we present detailed results for the double differential distributions, systematically varying the kinematical conditions. Section 4 contains a brief discussion of the underlying physics in relation to earlier studies and alternative mechanisms for pion emission.

\section{Analysis of the $2 \pi^{+} 2 \pi^{-}$data}

The reaction $\bar{p} p \rightarrow 2 \pi^{+} 2 \pi^{-}$at rest in the CPLEAR experiment proceeds from $S$ and $P$-wave atomic states [20]. The corresponding pion distribution for the final state

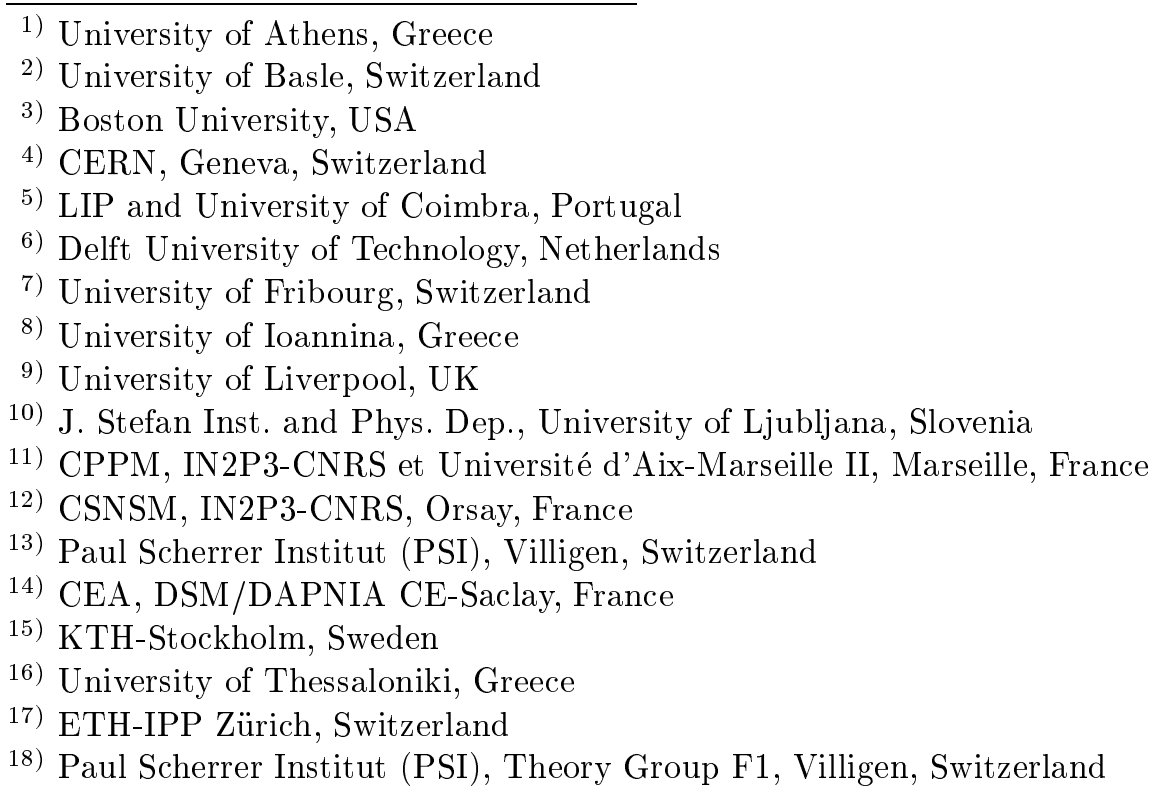


configuration $\left\{\mathbf{p}_{i}\right\}, i=1,2,3,4$, has the form

$$
d \sigma\left(\left\{\mathbf{p}_{i}\right\}\right) \sim\left|T\left(\mathbf{k},\left\{\mathbf{p}_{i}\right\}\right)\right|_{\mathbf{k} \rightarrow 0}^{2} d \Phi_{4}\left(p, p_{1}, p_{2}, p_{3}, p_{4}\right) .
$$

Here $T\left(\mathbf{k},\left\{\mathbf{p}_{i}\right\}\right)$ is the amplitude of the $\bar{p} \mathrm{p} \rightarrow 2 \pi^{+} 2 \pi^{-}$annihilation from the initial $\bar{p}$ p state with relative momentum $\mathbf{k}, d \Phi_{4}\left(p, p_{1}, p_{2}, p_{3}, p_{4}\right)$ is the four-particle relativistic phase space, and the limit $\mathbf{k} \rightarrow 0$ implies the incoherent addition of the $S$ - and $P$-wave annihilation occurring in the experiment. The four-vectors in Eq. (1) are $p_{i}=\left(E_{i}, \mathbf{p}_{i}\right)$, and $p=$ $\left(2 m_{p}, 0\right)$, the total four-momentum for $\bar{p} p$ annihilation at rest. The notation implies a sum over initial spin states, and all quantum numbers specifying the initial spin state are suppressed. In this paper we shall determine $\left|T\left(\mathbf{k},\left\{\mathbf{p}_{i}\right\}\right)\right|$ for each event directly.

\subsection{Event selection}

The CPLEAR detector [21] is cylindrically symmetric and placed inside a solenoidal magnet of $3.6 \mathrm{~m}$ length and $2 \mathrm{~m}$ diameter, with a field of $0.44 \mathrm{~T}$. Antiprotons of 200 $\mathrm{MeV} / \mathrm{c}$ momentum, provided by LEAR, stop and annihilate at the centre of the detector, in a spherical target of $7 \mathrm{~cm}$ radius filled with gaseous hydrogen at 16 bar. Tracking is provided by two layers of proportional chambers, six layers of drift chambers, and two layers of streamer tubes. Outside the tracking devices there are 32 sectors of Scintillator (S1) - Cherenkov (C) - Scintillator (S2) sandwiches providing particle identification. The outermost detector is an 18-layer gas-sampling electromagnetic calorimeter.

The data analysed here represent a small fraction of the CPLEAR data and were collected with the so-called minimum-bias trigger in 1993 and $1994{ }^{1)}$. This trigger requires a hit in the scintillator S1 in coincidence with the incoming antiproton and accepts events in the entire phase space, limited only by the energy thresholds and geometrical acceptances. From the total of about $5.5 \cdot 10^{7}$ minimum-bias events, well balanced between opposite magnetic-field polarity settings, $10^{7}$ four-prong events were selected according to the following criteria:

1. four tracks balanced in charge;

2. good quality of track reconstruction (minimal number of tracking hits, good $\chi^{2}$ for the track fit) and the vertex coordinates of all track pairs are inside the target sphere.

To select exclusively the events of the $2 \pi^{+} 2 \pi^{-}$channel, kinematical and topological cuts were applied:

1. the $4 \pi$ invariant mass $m_{4 \pi}$, measured with a resolution of $\sigma\left(m_{4 \pi}\right)=0.05 \mathrm{GeV}$, is required to be consistent with $2 m_{p}\left(\left|m_{4 \pi}-2 m_{p}\right|<0.08 \mathrm{GeV}\right)$ and the missing energy to be very small (less than the $\pi^{0}$ mass);

2. the momentum of each track (pion) must be in the range $0.06 \leq p_{\pi} \leq 0.92 \mathrm{GeV} / c$;

3. an opening angle $\geq 60 \mathrm{mrad}$ between any two charged tracks is required to avoid pion pairs with insufficient two-track resolution and lepton pairs from $\gamma$ conversion and $\pi^{0}$ Dalitz decays.

A total of $4.2 \cdot 10^{5} 2 \pi^{+} 2 \pi^{-}$events remained, characterized by very little missing energy and negligible kaon contamination (a big advantage over many previous pion correlation studies). With a single-track momentum resolution of about 5\%, a resolution of

1) The data published earlier in Ref. [8] were collected in 1991 and 1992 with the same trigger. 
$\sigma\left(M^{2}\right) \leq 0.005 \mathrm{GeV}^{2}$ is achieved in the region of interest (low $M^{2}$ ). A typical bin width is $0.02 \mathrm{GeV}^{2}$.

The measured pion momentum distributions for $\pi^{+}$and $\pi^{-}$are plotted in Fig. 1. The perfect agreement between these two spectra shows that there are no systematic differences. The shape of the momentum distribution is quite close to phase space which is also shown in Fig. 1. The structure around $0.45 \mathrm{GeV}$ is from $\rho \rho$ events.

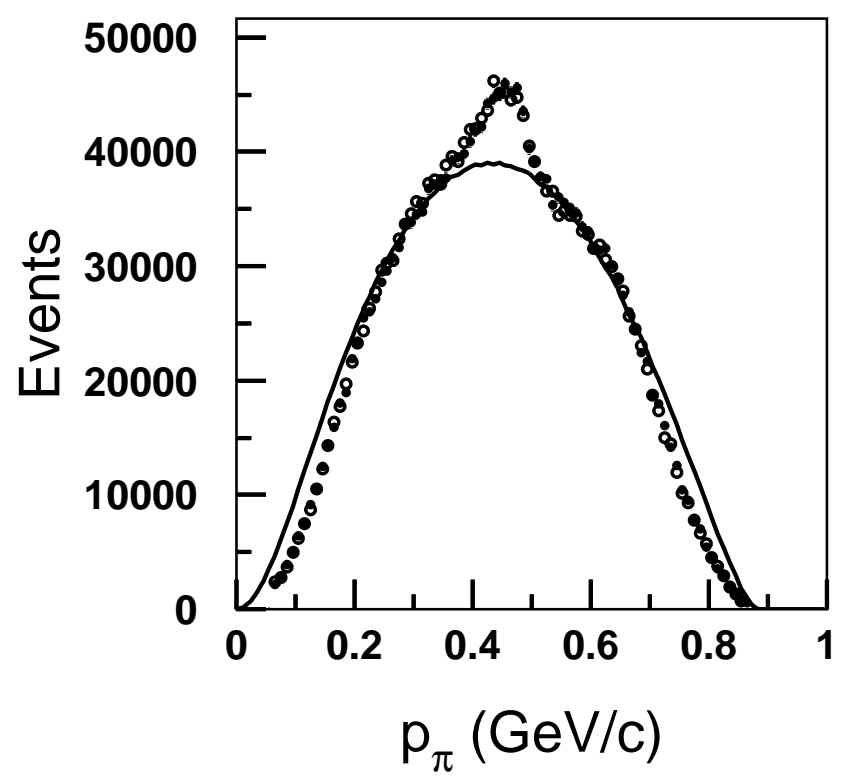

Figure 1: The measured single-pion momentum distribution $d N_{\pi} / d\left|\mathbf{p}_{\pi}\right|$ for the $2 \pi^{+} 2 \pi^{-}$ channel, values for $\pi^{+}(\bullet)$ and $\pi^{-}(\circ)$ are shown. The line is the phase-space distribution for the $2 \pi^{+} 2 \pi^{-}$channel.

\subsection{Correlation functions for inclusive distributions}

One of the definitions of pion pair correlations is based on the two-particle density in momentum space:

$$
c\left(p_{1}, p_{2}\right)=\rho_{2}\left(p_{1}, p_{2}\right)-\rho_{1}\left(p_{1}\right) \rho_{1}\left(p_{2}\right),
$$

where $\rho_{2}\left(p_{1}, p_{2}\right)$ is the two-particle inclusive density and $\rho_{1}\left(p_{1}\right)$ is the single-particle inclusive density. All observables not appearing explicitly are integrated out. The inclusive distributions are related to the differential cross-sections:

$$
\begin{aligned}
\rho_{1}\left(p_{1}\right) & =\sigma^{-1} \frac{d \sigma}{d^{3} \mathbf{p}_{1} / 2 E_{1}} \\
\rho_{2}\left(p_{1}, p_{2}\right) & =\sigma^{-1} \frac{d \sigma}{d^{3} \mathbf{p}_{1} /\left(2 E_{1}\right) d^{3} \mathbf{p}_{2} /\left(2 E_{2}\right)} .
\end{aligned}
$$


Alternatively the two-particle correlations can be described in terms of the ratio

$$
C\left(p_{1}, p_{2}\right)=\frac{\rho_{2}\left(p_{1}, p_{2}\right)}{\rho_{0}\left(p_{1}, p_{2}\right)},
$$

where $\rho_{0}\left(p_{1}, p_{2}\right)$ is the two-particle distribution in the absence of correlations, with various prescriptions being used in the literature. One choice, consistent with Eq. (2), is the product of the single-particle densities $\rho_{0}\left(p_{1}, p_{2}\right)=\rho_{1}\left(p_{1}\right) \rho_{1}\left(p_{2}\right)$. Another choice of reference sample mentioned in the introduction is to take the two-particle inclusive distribution for unlike pions: $\rho_{0}\left(p_{1}, p_{2}\right)=\rho_{2}^{+-}\left(p_{1}, p_{2}\right)$ [5-8]. In this case some experimental uncertainties cancel out in the ratio $R_{2}\left(p_{1}, p_{2}\right)=\rho_{2}^{++\mid--}\left(p_{1}, p_{2}\right) / \rho_{2}^{+-}\left(p_{1}, p_{2}\right)$. However, the correlation function thus calculated is expected to be distorted by the different dynamics for particles of unlike charge [5,22].

Averaging over angles and momenta, the correlation functions can be expressed as a function of one parameter, i.e. the two-pion invariant mass $M$ :

$$
\begin{aligned}
C(M) & =\frac{\rho_{2}(M)}{\left(\rho_{1} \cdot \rho_{1}\right)(M)} \\
\rho_{2}(M) & =\int \delta\left(M-\sqrt{\left(p_{1}+p_{2}\right)^{2}}\right) \rho_{2}\left(p_{1}, p_{2}\right) \frac{d^{3} \mathbf{p}_{1} d^{3} \mathbf{p}_{2}}{\left(2 E_{1}\right)\left(2 E_{2}\right)} \\
\left(\rho_{1} \cdot \rho_{1}\right)(M) & =\int \delta\left(M-\sqrt{\left(p_{1}+p_{2}\right)^{2}}\right) \rho_{1}\left(p_{1}\right) \rho_{1}\left(p_{2}\right) \frac{d^{3} \mathbf{p}_{1} d^{3} \mathbf{p}_{2}}{\left(2 E_{1}\right)\left(2 E_{2}\right)} .
\end{aligned}
$$

The invariant mass $M$ is uniquely related to the square of the momentum difference:

$$
\left(p_{1}-p_{2}\right)^{2}=4 \mu^{2}-M^{2}=-Q^{2},
$$

where $\mu$ is the pion mass and $\mathbf{Q}$ is the difference of the three-momenta of the two pions in their centre-of-mass system (CMS), therefore the variables $M^{2}$ and $Q^{2}$ are equivalent.

Because of the total energy-momentum conservation, the ratio $C(M)$ is not a constant even if the distributions $d \sigma /\left(d^{3} \mathbf{p}_{1} / 2 E_{1}\right)$ and $d \sigma /\left(d^{3} \mathbf{p}_{1} / 2 E_{1}\right)\left(d^{3} \mathbf{p}_{2} / 2 E_{2}\right)$ are determined by phase space alone. As long as the two particles carry a small fraction of the total energy, which is a typical situation for high-energy collisions, these effects are not significant. However, for the $\bar{p} p$ annihilation at rest, the dependence of $C(M)$ on $M$ is significant even for the pure phase-space distribution, as shown in Fig. 2 for the annihilation into four pions. It is clear that these kinematical correlations must be removed from the correlation function $C(M)$ to study the dynamics of the pion production.

\subsection{Single-variable two-pion correlations}

We present here the single-variable two-pion correlations $R_{2}(M)$ and $C(M)$ which have been frequently used in previous analyses. In order to isolate the correlation effects we compare the experimental density with a four-pion phase-space distribution corrected for experimental cuts and efficiencies in the same way as the data.

The data sample of $4.2 \cdot 10^{5}$ events was used to calculate the two-particle distributions $\rho_{2}(M)$ defined by Eq. (7) for pairs of identical pions, $\rho_{2}^{++}(M)$ and $\rho_{2}^{--}(M)$, and pairs of unlike pions ${ }^{2)}, \rho_{2}^{+-}(M)$. The corresponding two-particle density from the phase-space

2) Here and below all distributions for unlike-pion pairs contain multiple entries per event corresponding to all possible $\pi^{+} \pi^{-}$combinations. 

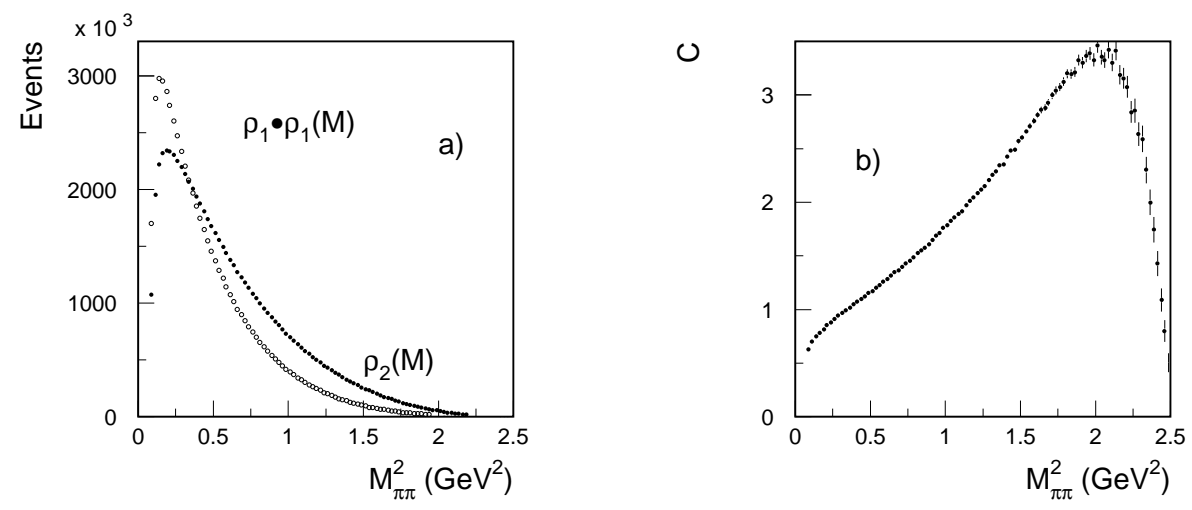

Figure 2: (a) The calculated invariant-mass distributions $\rho_{2}(M)$ and $\left(\rho_{1} \cdot \rho_{1}\right)(M)$ and (b) the correlation function $C(M)$ for $\overline{\mathrm{p} p} \rightarrow 4 \pi$ at $\sqrt{s}=2 m_{p}$, assuming a pure phase-space distribution. The error bars shown are the statistical errors of the phase-space integration.

simulation is called $\rho_{2}^{P S}(M)$. In Figs.3a and 3b we consider the ratios of distributions for like- and unlike-pion pairs normalized to phase space, $\rho_{2}^{++|--|+-}(M) / \rho_{2}^{P S}(M)$, for which the kinematical correlations discussed in Section 2.2 cancel. Figure $3 c$ shows the ratio of experimental two-particle distributions

$$
R_{2}(M)=\frac{\rho_{2}^{++\mid--}(M)}{\rho_{2}^{+-}(M)}
$$

The results for $R_{2}(M)$ are consistent with those previously reported [8]. Comparing Figs. $3 \mathrm{a}$ and $3 \mathrm{~b}$ one sees that the peak in $R_{2}(M)$ is mostly due to the strong depletion of the unequal charge distribution at small $Q^{2}$ which is due to the presence of $\rho$ mesons and other resonances, absent in the $\pi^{+} \pi^{+}$and $\pi^{-} \pi^{-}$channels. It is therefore dangerous to deduce model parameters from the ratio $R_{2}(M)$, as has been discussed many times $[4,9,22]$.

To study the correlation function $C(M)$ in Eq. (6), the two-particle distribution for uncorrelated pion pairs was calculated using the event-mixing method. In this method two particles are selected from two different events of the experimental data set, the invariant mass of the pair is calculated, and the corresponding distribution $\left(\rho_{1} \cdot \rho_{1}\right)(M)$ is generated. The complete data sample was used in this procedure, i.e. no extra cuts were applied ${ }^{3)}$. The experimental distribution $\left(\rho_{1} \cdot \rho_{1}\right)(M)$ normalized to phase space (see below) is plotted in Fig. 3d. Separate $\left(\rho_{1} \cdot \rho_{1}\right)(M)$ distributions were analysed for ,++-- , and +- pion pairs and all found to be consistent. We have checked that the direct evaluation of $\left(\rho_{1} \cdot \rho_{1}\right)(M)$ using the experimental single-particle density $\rho_{1}(p)$ leads to consistent results. The ratio shown in Fig. $3 \mathrm{~d}$ is fairly flat at small $Q^{2}$. The small structure seen for $Q^{2}<0.2 \mathrm{GeV}^{2}$ is not physical. Figures $4 \mathrm{a}$ and $4 \mathrm{~b}$ show the correlation function $C(M)$. In order to account for the trivial $M$-dependence which arises because of to the energy-momentum conservation for the pure phase-space distribution (see Fig. 2) and from the experimental cuts, the following double ratios were calculated (see Figs. 4c

3) Note that the event mixing in the analysis of high-energy collisions usually involves further selection criteria, see for example [13]. 


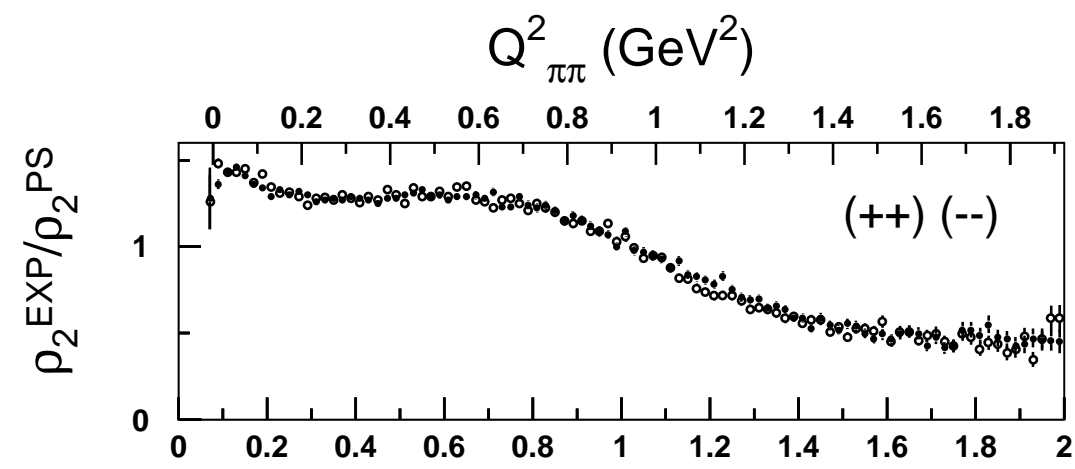

a)

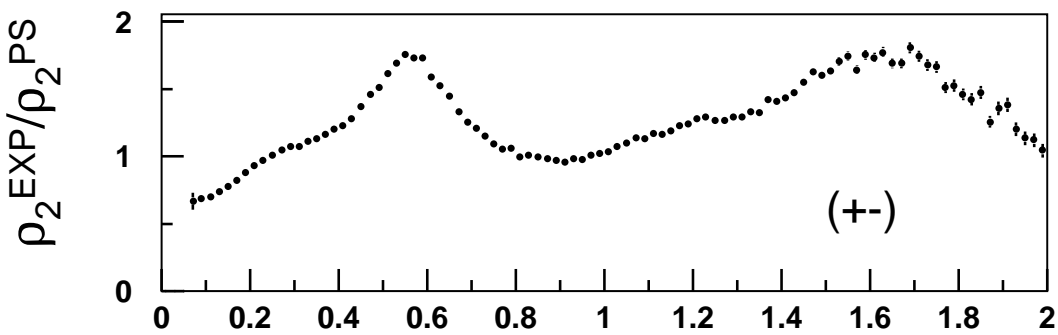

b)

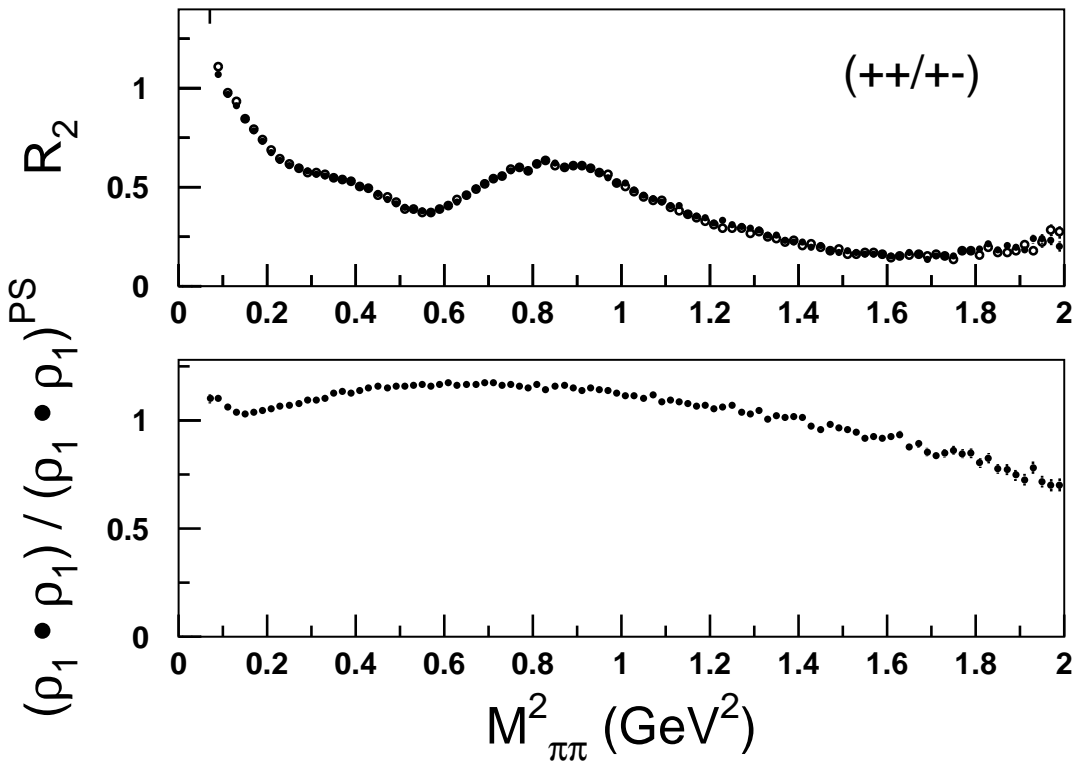

c)

d)

Figure 3: Experimental two-particle distributions $\rho_{2}(M)$, Eq. (7), divided by phase space $\rho_{2}^{P S}(M)$ for (a) pairs of identical pions, $\pi^{+} \pi^{+}(\bullet)$ and $\pi^{-} \pi^{-}(\mathrm{o})$, and (b) pairs of unlike pions. (c) The ratio $R_{2}$ of the experimental two-particle distributions for like and unlike pions: $\rho_{2}^{++}(M) / \rho_{2}^{+-}(M)(\bullet)$ and $\rho_{2}^{--}(M) / \rho_{2}^{+-}(M)(\circ)$. (d) The ratio of the experimental distribution $\left(\rho_{1} \cdot \rho_{1}\right)(M)$ to the phase-space distribution $\left(\rho_{1} \cdot \rho_{1}\right)^{P S}(M)$. 
and $4 d)$ :

$$
\frac{C^{++|--|+-}(M)}{C^{P S}(M)}=\frac{\rho_{2}^{++|--|+-}(M)}{\left(\rho_{1} \cdot \rho_{1}\right)(M)}: \frac{\rho_{2}^{P S}(M)}{\left(\rho_{1} \cdot \rho_{1}\right)^{P S}(M)} .
$$

Note that the distributions for like and unlike pairs in Figs. 4c and 4d look similar to the corresponding distributions shown in Figs. 3a and 3b except for normalization. The like-sign correlation function in Fig. 4c shows a weak peaking at small $Q^{2}$, but the signal is not strong enough for a meaningful analysis. We shall show in the next section that the weakness of the signal is due to the integration over the mass of the second pion pair in phase space.

\section{Differential two-pion correlations}

So far we presented results for the inclusive correlation function $C^{++\mid--}(M) / C^{P S}(M)$ where all kinematical variables except $M$ have been integrated out. The correlation signal can be isolated by considering a double differential density. We introduce the two-pion subsystems $a$ and $b$ with four-momenta $p_{a}=\left(p_{1}+p_{2}\right)$ and $p_{b}=\left(p_{3}+p_{4}\right)$ and invariant masses $M_{a}$ and $M_{b}$. Integrating over the angles specifying the relative orientation of the momenta in the final state we define the double differential cross section:

$$
\frac{d \sigma}{d M_{a}^{2} d M_{b}^{2}} \sim W\left(s, M_{a}, M_{b}\right) \int\left|T\left(\mathbf{k},\left\{\mathbf{p}_{i}\right\}\right)\right|_{\mathbf{k} \rightarrow 0}^{2} d \Omega_{a b} d \Omega_{12} d \Omega_{34}
$$

where the factor $W\left(s, M_{a}, M_{b}\right)$ is given by

$$
\begin{aligned}
W\left(s, M_{a}, M_{b}\right) & =\frac{P_{a b}}{\sqrt{s}} \sqrt{\left(1-\frac{4 \mu^{2}}{M_{a}^{2}}\right)\left(1-\frac{4 \mu^{2}}{M_{b}^{2}}\right)}, \\
P_{a b} & =\sqrt{\frac{\left(s-\left(M_{a}+M_{b}\right)^{2}\right)\left(s-\left(M_{a}-M_{b}\right)^{2}\right)}{4 s}} .
\end{aligned}
$$

Here $P_{a b}$ is the relative three-momentum of the two-pion pairs. Removing the phase-space factor $W\left(s, M_{a}, M_{b}\right)$ we define the double differential density:

$$
\begin{aligned}
\varrho\left(M_{a}, M_{b}\right) & =\frac{1}{W\left(s, M_{a}, M_{b}\right)} \frac{d \sigma}{d M_{a}^{2} d M_{b}^{2}} \\
& \sim \int\left|T\left(\left\{\mathbf{p}_{i}\right\}\right)\right|^{2} d \Omega_{a b} d \Omega_{12} d \Omega_{34} .
\end{aligned}
$$

Note that the pair of variables $\left(M_{a}, M_{b}\right)$ can be replaced by $\left(Q, P_{a b}\right)$, where the $Q$ of Eq. (9) is twice the relative three-momentum between the pions 1 and 2 in their centre-of-mass system and the relative momentum $P_{a b}$ is equal to the total momentum, $P_{a b}=\left|\mathbf{p}_{1}+\mathbf{p}_{2}\right|$, in the overall CMS. Note also that, contrary to $\rho_{2}\left(M_{a}\right) \sim d \sigma / d M_{a}$ of Eq. (7), the differential density $\varrho\left(M_{a}, M_{b}\right)$ is a constant if the relativistic phase-space approximation is valid, i.e. if the matrix element $T$ has no momentum dependence.

Figure 5 shows the physical region in the $\left(M_{a}^{2}, M_{b}^{2}\right)$ plane for the reaction $\bar{p} p \rightarrow 4 \pi$ at rest, with the contour plot displaying the values of $P_{a b}^{2}$. The region of small $Q^{2}$ where symmetrization effects are expected is in the lower left corner.

Differential cross-sections and densities for equal-charge pairs are shown in Fig. 6. There is a strong enhancement as both invariant masses approach threshold: $M_{++}^{2}, M_{--}^{2} \rightarrow$ $4 \mu^{2}$. This peaking occurs for a large relative momentum $P_{a b}$ between the two-pion pairs. 


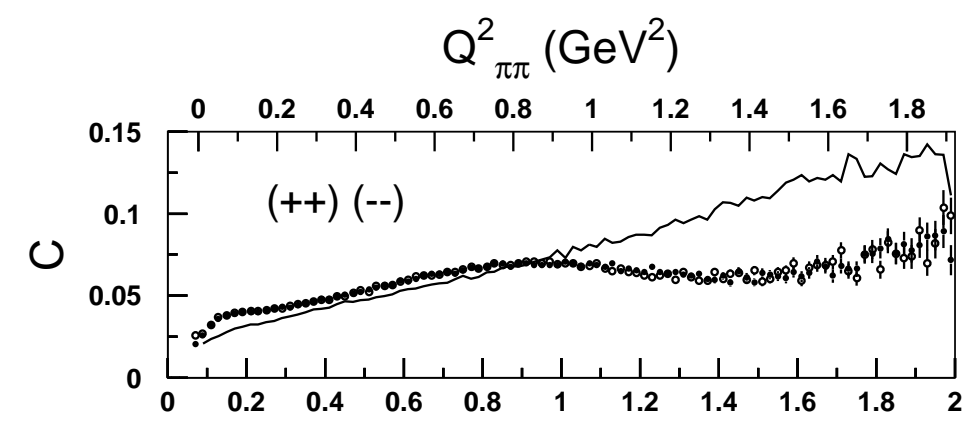

a)

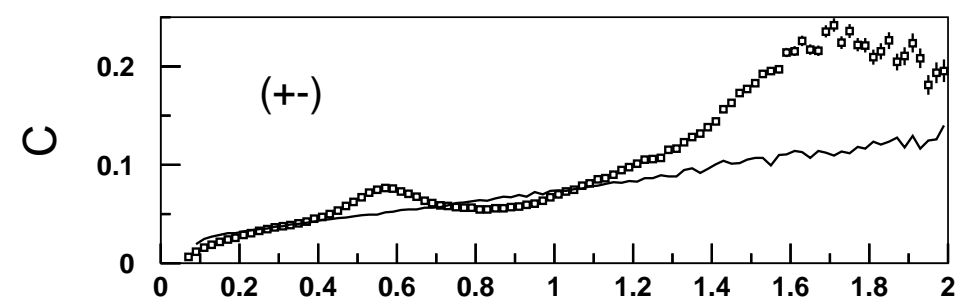

b)

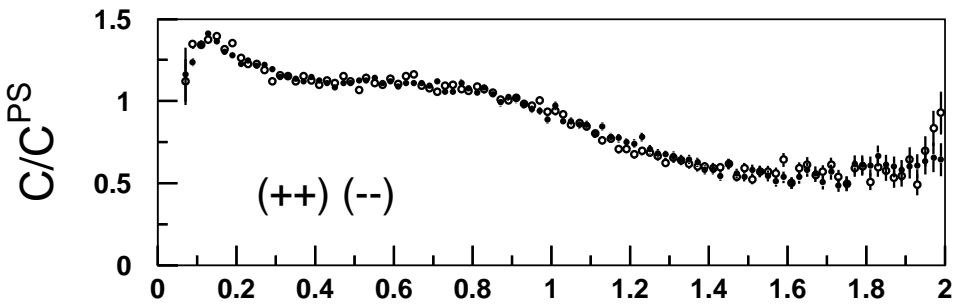

c)

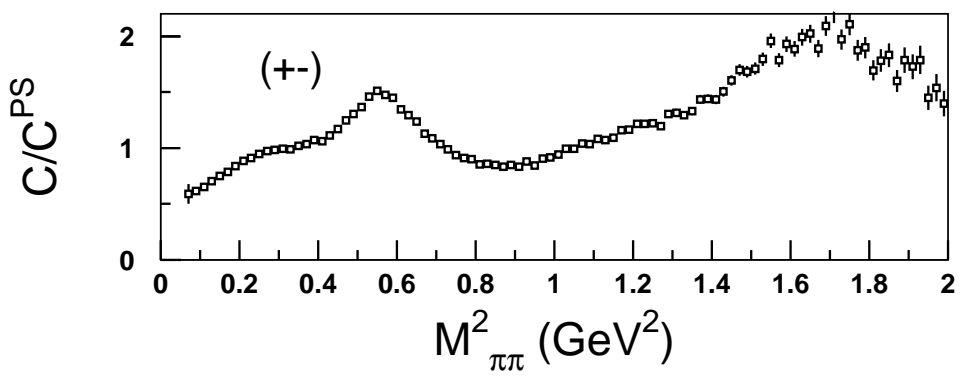

d)

Figure 4: The experimental correlation function $C(M)$, Eq. (6), vs. the square of the invariant mass of two particles $M^{2}$ : (a) $\rho_{2}^{++}(M) /\left(\rho_{1} \cdot \rho_{1}\right)(M)(\bullet)$ and $\rho_{2}^{--}(M) /\left(\rho_{1} \cdot \rho_{1}\right)(M)$ (o), (b) $\rho_{2}^{+-}(M) /\left(\rho_{1} \cdot \rho_{1}\right)(M)(\square)$. The lines in (a) and (b) show the corresponding phasespace ratio $\rho_{2}^{P S}(M) /\left(\rho_{1} \cdot \rho_{1}\right)^{P S}(M)$. The experimental correlation functions normalized to the phase-space distribution, $C(M) / C^{P S}(M)$, are shown in (c) for $\pi^{+} \pi^{+}(\bullet)$ and $\pi^{-} \pi^{-}$ (o), and in (d) for $\pi^{+} \pi^{-}$. 


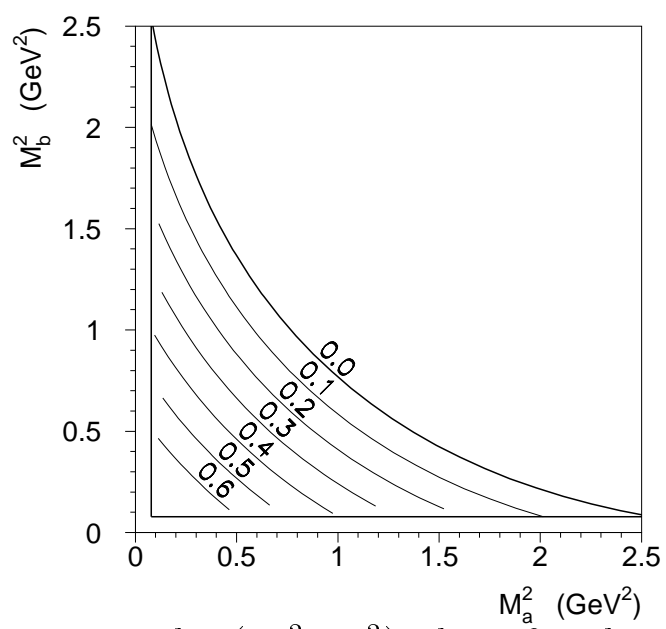

Figure 5: The physical region in the $\left(M_{a}^{2}, M_{b}^{2}\right)$ plane for the reaction $\bar{p} p \rightarrow 4 \pi$ at $\sqrt{s}=$ $2 m_{p}$. The contour plot shows the values of $P_{a b}^{2}$ in $\mathrm{GeV}^{2}$.

The differential density $\varrho\left(M_{+-(a)}, M_{+-(b)}\right)$ for the unlike-pion pairs with the invariant masses $M_{+-(a)}$ and $M_{+-(b)}$ is plotted in Fig. 7. Contrary to the case of the like-pion pairs, there is no significant enhancement at small $M_{+-}^{2}$ in this distribution. A characteristic feature of $\varrho\left(M_{+-(a)}^{2}, M_{+-(b)}^{2}\right)$ is the strong signal from $\rho$ mesons.

In order to check that the $\rho$ mesons do not simulate pion correlation signals for likecharge pion pairs at small $Q^{2}$ we show in Fig. 8 double differential densities for the events where $\rho \rho$ configurations are removed, $\varrho^{n o} \rho \rho\left(M_{++}, M_{--}\right)$, and for the events exclusively from $\rho \rho$ configurations, $\varrho^{\text {only } \rho \rho}\left(M_{++}, M_{--}\right)$. The $\rho$-meson pairs have been defined by the following condition for the unlike-pion pairs $a$ and $b$ :

$$
\left(M_{a}^{2}-m_{\rho}^{2}\right)+\left(M_{b}^{2}-m_{\rho}^{2}\right)<2.2 m_{\rho} \Gamma_{\rho}
$$

where $m_{\rho}$ and $\Gamma_{\rho}$ are the mass and the width of the $\rho$ meson. The suppression of the $\rho \rho$ events is made incoherently; there are $1.34 \cdot 10^{5}$ events ( $46 \%$ of the total) outside the $\rho \rho$ region. As shown in Fig. 8 a, the $\rho \rho$ states do not produce the enhancement at small $M_{++}$ and $M_{--}$, which is therefore not due to kinematical reflections from the $\rho \rho$ channel.

In order to verify that the enhancement in $\varrho\left(M_{++}, M_{--}\right)$at $M_{++}^{2}, M_{--}^{2} \rightarrow 4 \mu^{2}$ is not produced by kinematical reflections from any other resonance channels we used a Monte Carlo simulation for the following channels with four pions in the final state: $\rho^{0} \rho^{0} \rightarrow$ $2 \pi^{+} 2 \pi^{-}, \rho^{0} \pi^{+} \pi^{-} \rightarrow 2 \pi^{+} 2 \pi^{-}, a_{2}^{ \pm} \pi^{\mp} \rightarrow \rho^{0} \pi^{+} \pi^{-} \rightarrow 2 \pi^{+} 2 \pi^{-}, f_{2} \pi^{+} \pi^{-} \rightarrow 2 \pi^{+} 2 \pi^{-}$. The events were generated with the CPLEAR simulation program and subsequently passed through the same analysis criteria as real data. The results are plotted in Fig. 9 and show no significant enhancement in the region of interest.

It is known that the resonant mechanisms $\rho^{0} \pi^{+} \pi^{-} \rightarrow 2 \pi^{+} 2 \pi^{-}$and $a_{2}^{ \pm} \pi^{\mp} \rightarrow \rho^{0} \pi^{+} \pi^{-} \rightarrow$ $2 \pi^{+} 2 \pi^{-}$dominate the $S$-wave $\bar{p}$ p annihilation at rest into $2 \pi^{+} 2 \pi^{-}[25,26]$. The Monte Carlo results shown in Fig. 9 do not take the amplitude symmetrization into account. In order to investigate possible effects of the symmetrization we performed calculations using a properly symmetrized amplitude for $\overline{\mathrm{p}} \mathrm{p}\left({ }^{3} S_{1}\right) \rightarrow \rho^{0}\left(\pi^{+} \pi^{-}\right)_{L=0} \rightarrow 2 \pi^{+} 2 \pi^{-}$. A moderate enhancement in the differential density $\varrho\left(M_{++}, M_{--}\right)$was found for this mechanism at small values of $M_{++}$and $M_{--}$. This enhancement, however, is significantly smaller than the one seen in the experimental data. The amplitude corresponding to the 

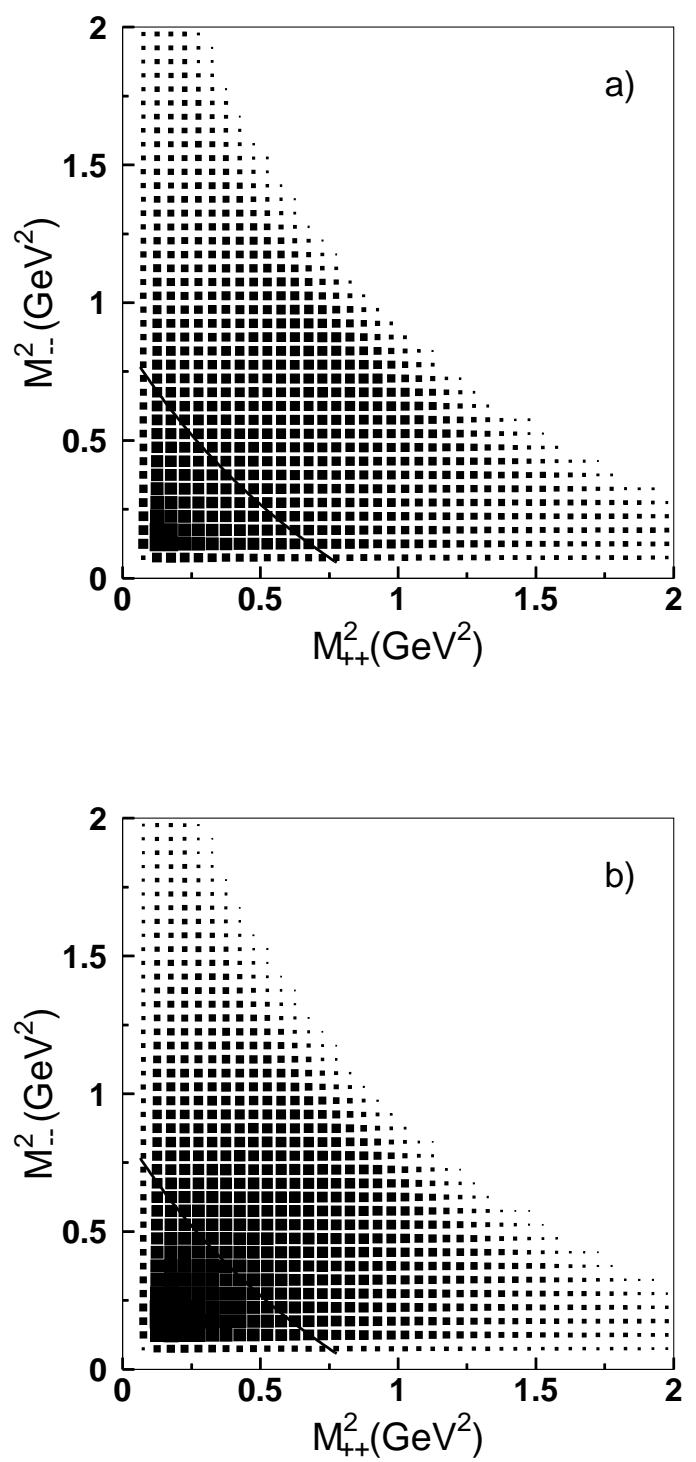

Figure 6: (a) The differential density $\varrho\left(M_{++}, M_{--}\right)$and (b) the differential cross-section $d \sigma / d M_{++}^{2} d M_{--}^{2} v s$. the invariant-masses squared of the like-pion pairs. The solid line shows the boundary $P_{a b}=0.7 \mathrm{GeV}$. (Note that in each figure the grey shades are renormalized to the same range of contrast.) 


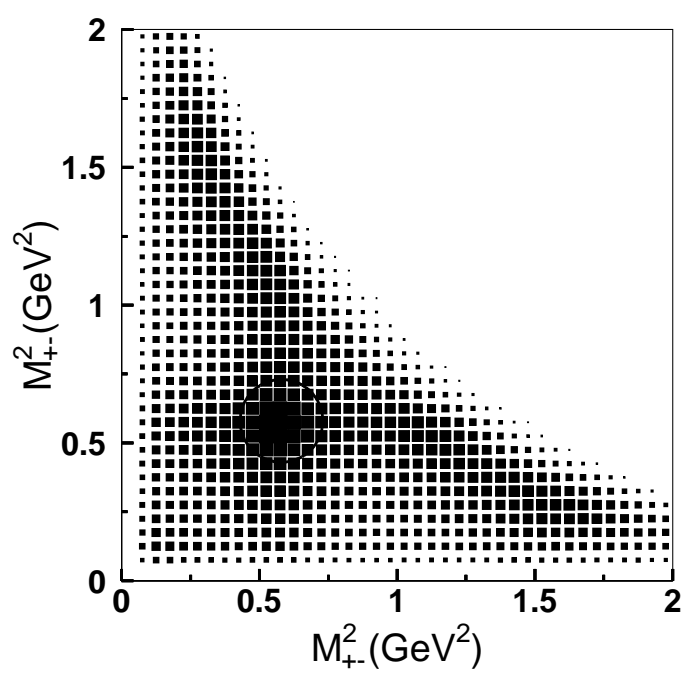

Figure 7: The differential density $\varrho\left(M_{+-(a)}, M_{+-(b)}\right)$ vs. the invariant-masses squared of the unlike-pion pairs. The circle shows the region defined by Eq. (17).

$a_{2}^{ \pm} \pi^{\mp} \rightarrow \rho^{0} \pi^{+} \pi^{-} \rightarrow 2 \pi^{+} 2 \pi^{-}$mechanism was found to be small for small values of $M_{++}$ and $M_{--}$.

For the further discussion in Section 4 we construct the following partial projections of the two-particle density. The two-dimensional space $\left(M_{++}^{2}, M_{--}^{2}\right)$ is divided into slices $M_{i}^{2} \leq M_{--\mid++}^{2}<M_{i+1}^{2}$ and the projections of $\varrho\left(M_{++}, M_{--}\right)$are defined by

$$
\varrho_{i}\left(M_{++\mid--}\right)=\int_{M_{i}^{2}}^{M_{i+1}^{2}} \frac{\varrho\left(M_{++}, M_{--}\right)}{\varrho^{P S}\left(M_{a}, M_{b}\right)} d M_{--\mid++}^{2} .
$$

The differential density $\varrho^{P S}\left(M_{a}, M_{b}\right)$ is calculated using the four-pion phase-space sample with the experimental cuts, where the pure phase-space distribution without these cuts would be a constant. The projections $\varrho_{i}\left(M_{++}\right)$are shown in Fig. 10. For small values of $M_{--}$, the peak in the projections $\varrho_{i}\left(M_{++}\right)$at $M_{++}^{2} \rightarrow 4 \mu^{2}$ is strongly enhanced in comparison with the inclusive correlation function in Fig. 4c. The corresponding results for $\varrho_{i}\left(M_{--}\right)$are consistent.

\section{Discussion}

One of the original motivations for studying pion pair correlations in annihilation at rest and in high-energy reactions is the HBT model [1], which was originally developed for light emission from a star. When applied to pion production $[2,4]$ it is assumed that pions are emitted from a finite-size source with a random phase at each emitting point. The chaoticity of the phase is critical for the standard argument [2,4], leading to the HBT enhancement of pion pair emission near $Q^{2}=0$. The slope of the correlation function is linked to the source radius $R$, and the relative strength of the peak $\lambda$ reflects the phase chaoticity ( $\lambda=0$ for coherent pion emission, $\lambda=1$ for fully incoherent emission). Since in proton-antiproton annihilation the signal in $C(M)$ is weak, we restrict the analysis to the partially projected distributions $\varrho_{i}(M)$, using a Gaussian parametrization for each slice $i$,

$$
\varrho_{i}(M)=N_{i}\left(1+\lambda_{i} \exp \left(-R_{i}^{2} Q^{2}\right)\right) .
$$



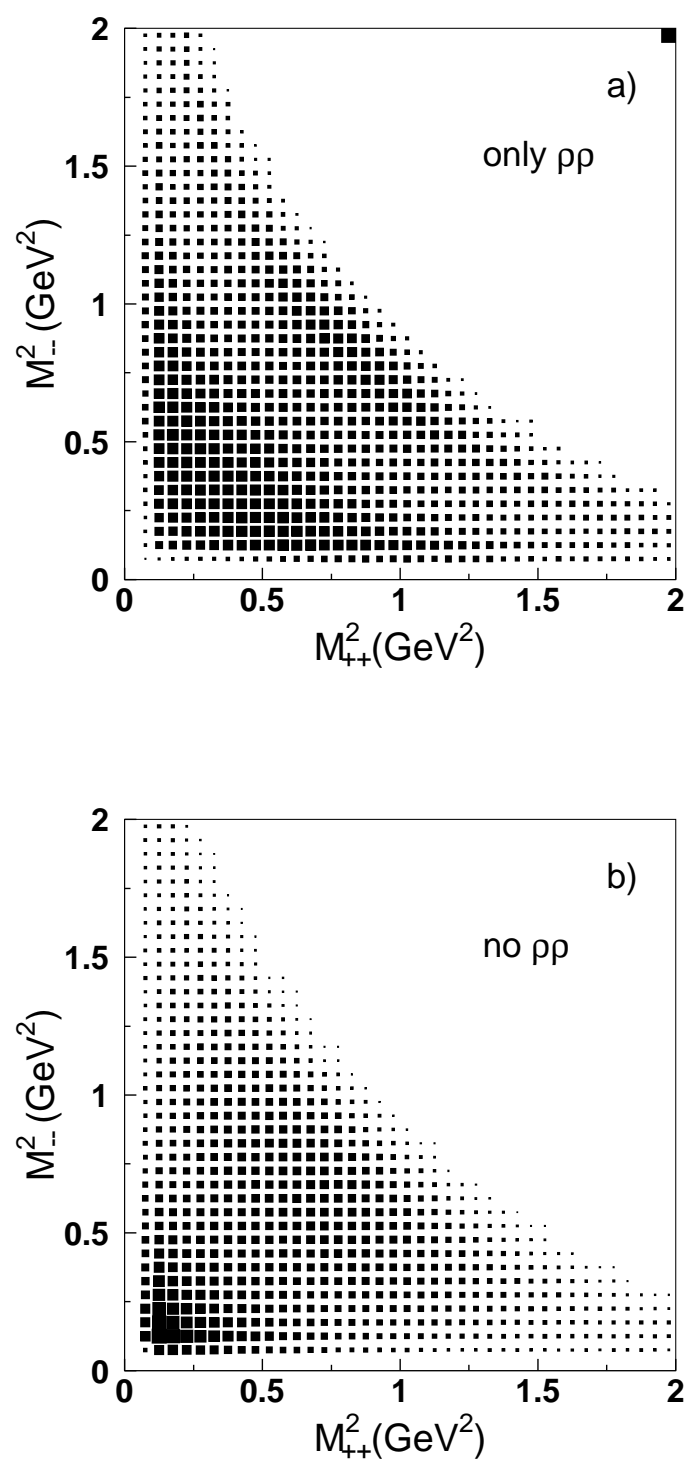

Figure 8: The differential densities (a) $\varrho^{\text {only } \rho \rho}\left(M_{++}, M_{--}\right)$and (b) $\varrho^{\text {no } \rho \rho}\left(M_{++}, M_{--}\right)$for the events including only $\rho \rho$ pairs (see Eq. (17) and Fig. 7) and excluding all the $\rho \rho$ pairs, respectively. 

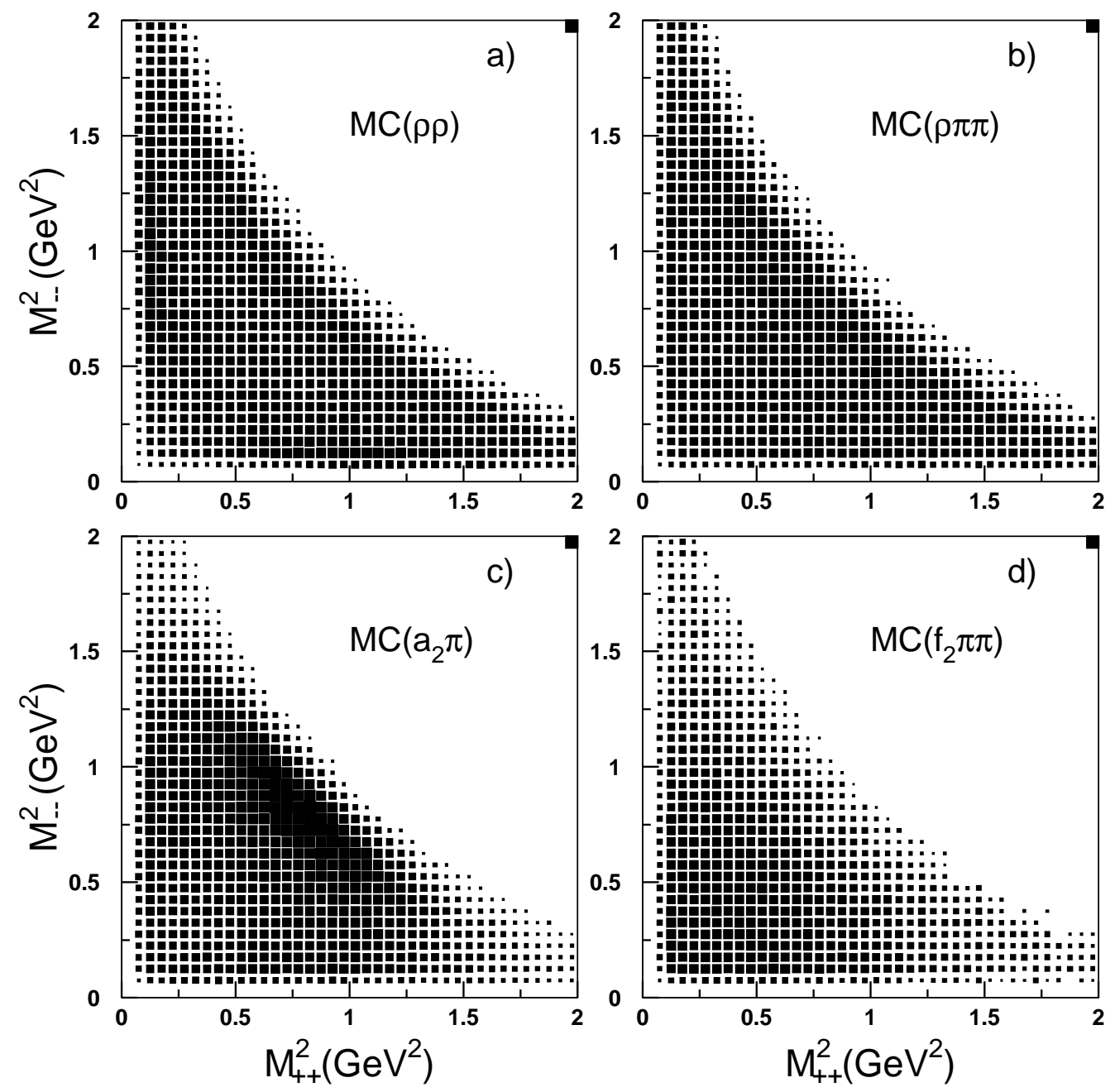

Figure 9: The differential density $\varrho\left(M_{++}, M_{--}\right)$for the MC events corresponding to the resonance channels: (a) $\rho^{0} \rho^{0} \rightarrow 2 \pi^{+} 2 \pi^{-}$, (b) $\rho^{0} \pi^{+} \pi^{-} \rightarrow 2 \pi^{+} 2 \pi^{-}$, (c) $a_{2}^{ \pm} \pi^{\mp} \rightarrow \rho^{0} \pi^{+} \pi^{-} \rightarrow$ $2 \pi^{+} 2 \pi^{-}$, (d) $f_{2} \pi^{+} \pi^{-} \rightarrow 2 \pi^{+} 2 \pi^{-}$. 


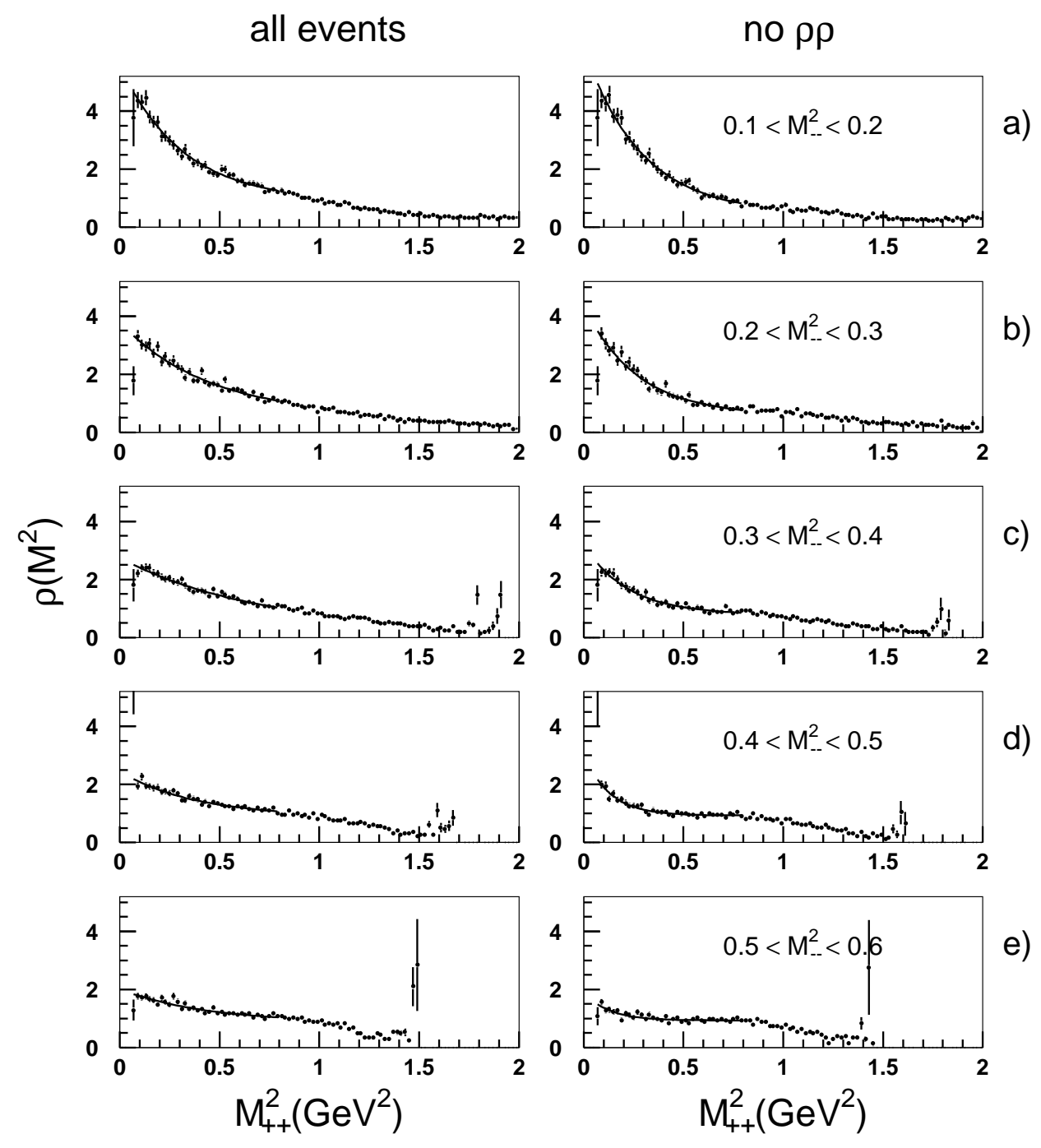

Figure 10: The projections $\varrho_{i}\left(M_{++}\right)$of $\varrho\left(M_{++}, M_{--}\right)$for different intervals of $M_{--}^{2}$ as indicated in the figure. The plots on the left correspond to Fig. 6a (all events) and the plots on the right to Fig. 8b ( $\rho \rho$ excluded). The lines correspond to the fit in Table 1. 
The values of $R$ and $\lambda$ resulting from fitting the projections in Fig. 10 are shown in Table 1. The fit was performed for both $\pi^{+} \pi^{+}$and $\pi^{-} \pi^{-}$correlations, and the results are consistent within the error bars. Shown also are the fits where the events from the kinematical region of the $\rho \rho$ pairs are excluded from the data completely.

The value for the source size $R$ depends rather weakly on the projection for $M_{--}^{2}<$ $0.6 \mathrm{GeV}^{2}$ which is a reasonable cut-off. The strength $\lambda$ decreases with increasing $M_{--}^{2}$ since the correlation signal in $\varrho_{i}\left(M_{++}\right)$is strongest for the projection corresponding to the lowest range of $M_{--}$. As mentioned earlier, this enhancement occurs at a large relative momentum $P_{a b}$ between the two-pion pairs.

Table 1: The radius $R$ and the strength $\lambda$ of the $\pi^{+} \pi^{+}$and $\pi^{-} \pi^{-}$correlations obtained from a fit of the partially projected distributions $\varrho_{i}(M)$ to Gaussian shape, Eq. (19). The mass interval for the first pion pair is indicated in the table; the fit was performed in the mass interval for the second pair $4 \mu^{2} \leq M^{2} \leq 0.8 \mathrm{GeV}^{2}$.

\begin{tabular}{|c|c|c|c|c|}
\hline \multicolumn{5}{|c|}{$\pi^{+} \pi^{+}$correlations } \\
\hline $\begin{array}{c}M_{--}^{2} \text { interval } \\
\left(\mathrm{GeV}^{2}\right)\end{array}$ & $\begin{array}{c}R \\
(\mathrm{fm})\end{array}$ & $\lambda$ & $N$ & $\chi^{2} / N D F$ \\
\hline \multicolumn{5}{|c|}{ all events } \\
\hline$(0.1,0.2)$ & $0.38 \pm 0.02$ & $3.7 \pm 0.5$ & $1.07 \pm 0.13$ & $14.0 / 11$ \\
\hline$(0.2,0.3)$ & $0.30 \pm 0.01$ & $4.0 \pm 0.6$ & $0.67 \pm 0.08$ & $12.1 / 11$ \\
\hline$(0.3,0.4)$ & $0.28 \pm 0.01$ & $3.1 \pm 0.5$ & $0.66 \pm 0.08$ & $8.9 / 11$ \\
\hline$(0.4,0.5)$ & $0.36 \pm 0.03$ & $1.1 \pm 0.1$ & $1.08 \pm 0.07$ & $6.2 / 11$ \\
\hline$(0.5,0.6)$ & $0.33 \pm 0.04$ & $0.7 \pm 0.1$ & $1.07 \pm 0.08$ & $11.9 / 11$ \\
\hline \multicolumn{5}{|c|}{$\rho \rho$ excluded } \\
\hline$(0.1,0.2)$ & $0.39 \pm 0.02$ & $8.4 \pm 1.2$ & $0.57 \pm 0.01$ & $10.0 / 11$ \\
\hline$(0.2,0.3)$ & $0.40 \pm 0.02$ & $4.3 \pm 0.5$ & $0.68 \pm 0.02$ & $8.7 / 11$ \\
\hline$(0.3,0.4)$ & $0.46 \pm 0.02$ & $2.2 \pm 0.1$ & $0.88 \pm 0.01$ & $6.2 / 11$ \\
\hline$(0.4,0.5)$ & $0.56 \pm 0.04$ & $1.1 \pm 0.1$ & $1.01 \pm 0.01$ & $10.6 / 11$ \\
\hline$(0.5,0.6)$ & $0.68 \pm 0.17$ & $0.43 \pm 0.20$ & $1.04 \pm 0.04$ & $8.1 / 11$ \\
\hline \multicolumn{5}{|c|}{$\begin{array}{l}\pi^{-} \pi^{-} \text {correlations } \\
\end{array}$} \\
\hline $\begin{array}{c}M_{++}^{2} \text { interval } \\
\left(\mathrm{GeV}^{2}\right)\end{array}$ & $\begin{array}{c}R \\
(\mathrm{fm})\end{array}$ & $\lambda$ & $N$ & $\chi^{2} / N D F$ \\
\hline \multicolumn{5}{|c|}{ all events } \\
\hline$(0.1,0.2)$ & $0.40 \pm 0.02$ & $3.5 \pm 0.2$ & $1.14 \pm 0.08$ & $23.3 / 11$ \\
\hline$(0.2,0.3)$ & $0.32 \pm 0.02$ & $3.5 \pm 0.6$ & $0.78 \pm 0.10$ & $8.2 / 11$ \\
\hline$(0.3,0.4)$ & $0.33 \pm 0.02$ & $2.0 \pm 0.2$ & $0.92 \pm 0.08$ & $12.8 / 11$ \\
\hline$(0.4,0.5)$ & $0.33 \pm 0.03$ & $1.4 \pm 0.2$ & $0.96 \pm 0.08$ & $5.5 / 11$ \\
\hline$(0.5,0.6)$ & $0.37 \pm 0.04$ & $0.87 \pm 0.08$ & $1.10 \pm 0.06$ & $6.8 / 11$ \\
\hline \multicolumn{5}{|c|}{$\rho \rho$ excluded } \\
\hline$(0.1,0.2)$ & $0.41 \pm 0.01$ & $7.8 \pm 0.8$ & $0.62 \pm 0.07$ & $19.0 / 11$ \\
\hline$(0.2,0.3)$ & $0.42 \pm 0.02$ & $4.0 \pm 0.3$ & $0.76 \pm 0.05$ & $2.4 / 11$ \\
\hline$(0.3,0.4)$ & $0.52 \pm 0.02$ & $2.2 \pm 0.1$ & $0.96 \pm 0.03$ & $9.1 / 11$ \\
\hline$(0.4,0.5)$ & $0.56 \pm 0.04$ & $1.4 \pm 0.1$ & $0.99 \pm 0.02$ & $5.0 / 11$ \\
\hline$(0.5,0.6)$ & $0.66 \pm 0.09$ & $0.62 \pm 0.15$ & $1.03 \pm 0.02$ & $3.8 / 11$ \\
\hline
\end{tabular}

It is natural to obtain the largest effect for a situation where the space resolution 
$P_{a b}^{-1}$ is able to resolve the source radius $R$. In order to resolve a source of size $R \approx 0.5 \mathrm{fm}$ we expect that each pion in the pair should have momentum $q>1 / R \approx 0.4 \mathrm{GeV}$. This corresponds to $P_{a b} \approx 2 q>0.8 \mathrm{GeV}$, i.e. only a small corner of the $\left(M_{++}^{2}, M_{--}^{2}\right)$ plane is suitable for this purpose. However, our result shows that in this favourable kinematical region the strength $\lambda \approx 4$, while for the conventional HBT correlation one expects $0 \leq$ $\lambda \leq 1$. Problems with the strength parameter $\lambda$ for $\bar{p} p$ annihilation at rest in the HBT framework have been noted earlier [5-10]. Our results for the differential density cast further doubts on a naive interpretation based on the HBT mechanism.

The size of the pion emission source given in Table 1 is smaller than other determinations for $\overline{\mathrm{p}} \mathrm{p}$ annihilation at rest [5-10]. In particular, the single Gaussian fit [6] gives $R \approx 0.8 \mathrm{fm}$, and the double Gaussian fit $[7,8]$ gives two radii: $R_{1} \approx(0.7-1.0) \mathrm{fm}$ and $R_{2} \approx(1.7-2.4) \mathrm{fm}$. The main reason is that the double differential density in Eq. (15) used here allows the pion emission source to be probed with variable and controlled resolution determined by the total momentum of the pion pair. The previous determinations for the $\bar{p} p$ annihilation at rest were based on integrated distributions which included pion pairs with insufficient total momentum to resolve the small size of the source. Note also that in Refs. [5-8] the radii were determined from the ratio $R_{2}(M)$.

While the HBT model leads to peaking at small $Q^{2}$, the statement cannot be reversed, as has been noted repeatedly in the literature [4,9]. In fact an enhancement of the correlation function near $Q^{2}=0$ can be obtained without requiring chaoticity or thermodynamic elements in the annihilation mechanism. One example is the Skyrmioninspired ansatz of Ref. [23]. The basic annihilation process is assumed to be very fast and entirely coherent. Translating the findings of Ref. [24] into the notation used here for the annihilation into four pions, a peak is predicted for $C(M)$ near $Q^{2}=0$ which results from averaging over angles and the momentum $P_{a b}$. To achieve the required destructive oscillations $P_{a b}$ has to be large. This is exactly as observed in Fig. 10, since the slices with a smaller value of $M_{b}=M_{--}$correspond to larger $P_{a b}$. Again the observation of the peaking and its kinematic properties are not sufficient proof of the correctness of the underlying dynamic model. On the other hand the slope of the correlation peak near $Q^{2}=0$ is related to the spatial extension of the pion source in the Skyrmion model as well.

\section{Conclusion}

For $\bar{p} \mathrm{p} \rightarrow 2 \pi^{+} 2 \pi^{-}$at rest we have established that the like-sign pion pairs are correlated at small relative momentum (or small invariant mass). In the exclusive $2 \pi^{+} 2 \pi^{-}$ channel considered here the phase-space factor is eliminated event by event for the double differential invariant-mass distributions. The double differential density $\varrho\left(M_{++}, M_{--}\right)$ thus constructed shows a very clear enhancement at small values of $M_{++}$or $M_{--}$. It is definitely not due to the presence of any resonances in the $\pi^{+} \pi^{-}$channel. At the same time the signal is fairly weak for the single-variable correlation function $C(M)$ because of the integration over all phase space available for the second pion pair.

The interpretation of the observed correlation signal by a conventional stochastic HBT mechanism remains questionable. While the present results do not allow us to conclusively discriminate between different correlation mechanisms, further analysis of correlation functions beyond single-variable inclusive distributions may be useful for this purpose.

A similar study of the exclusive reaction $\bar{p} p \rightarrow 2 \pi^{+} 2 \pi^{-} \pi^{0}$ is under way. The preliminary results are similar to those of the $4 \pi$ annihilation channel discussed here. It would be interesting to do a similar analysis for the annihilation into four and five neutral pions. 


\section{Acknowledgements}

We would like to thank the CERN LEAR staff as well as the technical and engineering staff of our institutes for their support and co-operation. This work was supported by the following institutions: the French CNRS/Institut National de Physique Nucléaire et de Physique des Particules, the French Commissariat à l'Energie Atomique, the Greek General Secretariat of Research and Technology, the Netherlands Foundation for Fundamental Research on Matter (FOM), the Portuguese JNICT, the Ministry of Science and Technology of the Republic of Slovenia, the Swedish Natural Science Research Council, the Swiss National Science Foundation, the UK Particle Physics and Astronomy Research Council (PPARC), and the US National Science Foundation.

\section{References}

[1] R. Hanbury-Brown and R.Q. Twiss, Phil. Mag. 45 (1954) 633.

[2] G. Goldhaber et al., Phys. Rev. Lett. 3 (1959) 181; G. Goldhaber et al., Phys. Rev. 120 (1960) 300.

[3] G. Cocconi, Phys. Lett. B49 (1974) 459.

[4] G.I. Kopylov and M.Y. Podgoretskii, Sov. J. Nucl. Phys. 19 (1974) 215.

[5] M. Deutschmann et al., Nucl. Phys. B204 (1982) 333.

[6] S. Ahmad et al., ASTERIX Collaboration, in Proc. of the Fourth LEAR Workshop (Villars-sur-Ollon, 1987), eds. C. Amsler et al. (Harwood, Chur, 1988) p. 697.

[7] K. Sarigiannis et al., CPLEAR Collaboration, Nucl. Phys. A558 (1993) 43c.

[8] R. Adler et al., CPLEAR Collaboration, Z. Phys. C63 (1994) 541.

[9] M. Gaspero, Nucl. Phys. A588 (1995) 861.

[10] R. Adler et al., CPLEAR Collaboration, Phys. At. Nucl. 59 (1996) 1422.

[11] A. Breakstone et al., Phys. Lett. B162 (1985) 400.

[12] T. Alexopoulos et al., Phys. Rev. D48 (1993) 1931.

[13] P. Abreu et al., DELPHI Collaboration, Z. Phys. C63 (1994) 17.

[14] T. Akesson et al., Phys. Lett. B129 (1983) 269.

[15] T. Akesson et al., Phys. Lett. B187 (1987) 420; T. Akesson et al., Z. Phys. C36 (1987) 517.

[16] C. Albajar et al., Phys. Lett. B226 (1989) 410.

[17] N. Neumeister et al., Phys. Lett. B275 (1992) 186.

[18] N. Neumeister et al., Z. Phys. C60 (1993) 633.

[19] T.C. Awes et al., Z. Phys. C69 (1995) 67.

[20] R. Adler et al., CPLEAR Collaboration, Phys. Lett. B267 (1991) 154; R. Adler et al., CPLEAR Collaboration, Z. Phys. C65 (1995) 199.

[21] R. Adler et al., CPLEAR Collaboration, Nucl. Instrum. Methods A379 (1996) 76.

[22] H.Q. Song, B.S. Zou, M.P. Locher, J. Riedlberger, P. Truöl, Z. Phys. A342 (1992) 439.

[23] R.D. Amado, F. Cannata, J.-P. Dedonder, M.P. Locher, Yang Lu, Phys. Lett. B339 (1994) 201

[24] R.D. Amado, F. Cannata, J.-P. Dedonder, M.P. Locher, Yang Lu, Phys. Rev. C51 (1995) 1587.

[25] M. Cresti et al., in Proc. Int. Conf. Elementary Particles (Siena, 1963), Vol. 1, eds.

G. Bernardini and G. Puppi (Soc. Ital. Fis., Bologna, 1963) p. 263.

[26] A. Bettini et al., Nuovo Cim. 38 (1965) 1495. 